\title{
A Rare Case of Thymic Carcinoma
}

\author{
Farshad Anvari ${ }^{1}$, Arnold M. Schwartz ${ }^{2}$, Gregory Trachiotis ${ }^{1}$ \\ ${ }^{1}$ Department of Surgery, Division of Cardiothoracic Surgery, \\ The George Washington University, Washington, DC, USA \\ ${ }^{2}$ Department of Pathology, The George Washington University, Washington DC, USA \\ E-mail:gtrachiotis@mfa.gwu.edu \\ Received February 3, 2011; revised April 4, 2011; accepted April 18, 2011
}

\begin{abstract}
Thymic carcinomas are unusual tumors of the thymus gland. Basaloid carcinoma, an unusal epithelioid varient of a thymic carcinoma, is a rare histopathological subtype, and is not well charecterized in the literature. We present the anatomical and histogical features of a basoloid thymic carcinoma, and discuss current diagnosis and imaging strategies, as well as the operative and oncologic care of this type of thymmic carcinoma. Basaloid carcinomas of the thymus after complete surgical resection and adjuvant therapy genarally have a favorable long-term prognosis.
\end{abstract}

Keywords: Thymus; Thymic Carcinoma

\section{Introduction}

Thymomas are the second most common tumors of the mediastinum; however, they are relatively uncommon tumors and often discovered on imaging studies of the chest performed for other reasons [1]. Thymic carcinomas are even rarer, accounting for $10 \%$ if all thymic tumors, but they still remain a significant disease because of their invasiveness and behave biologically different than other tumors of the thymus gland [2-5].We report a case of basaloid thymic carcinoma, one of the rarest variants of thymic carcinomas, and discuss diagnostic, operative and oncologic management.

\section{Case Summary}

A 64 year old male with no significant past medical history presented to the emergency room with one day history of myalgia, arthralgia, vertigo and shortness of breath with chest pressure. The patient had been experiencing right sided chest pain not related to exercise. The patient had a normal physical examination, and specifically no neurologic defects and a normal testicular exam. The chest radiograph showed a widened mediastinum, and a cardiac evaluation was negative, and and chest computerized tomogram (chest CT) to evaluate for pulmonary embolus demonstrated an anterior medicastinal mass masuring $3.9 \mathrm{~cm} \times 5.9 \mathrm{~cm}$ (Figure 1). Serum markers including alpha-fetoprotein, human choriogo-

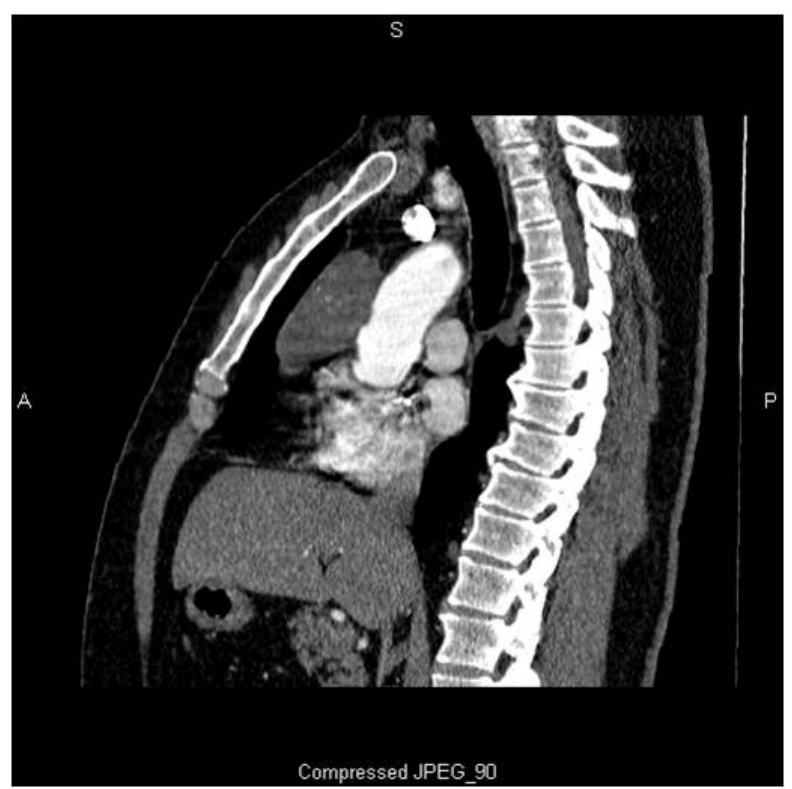

Figure 1. Chest computed tomography on admission showing an anterior mediastinal tumor.

nadotropin (HCG), CA-19-9, human immunodeficiency virus (HIV), and markers for myasthenia gravis were normal. A testicular ultrasound which was also obtained was negative. Preoperative cardiopulomonary testing was normal. The patient was scheduled for elective thymectomy. The exposure of the mediastinum via a sternotomy and revealed a large thymic mass grossly meas- 


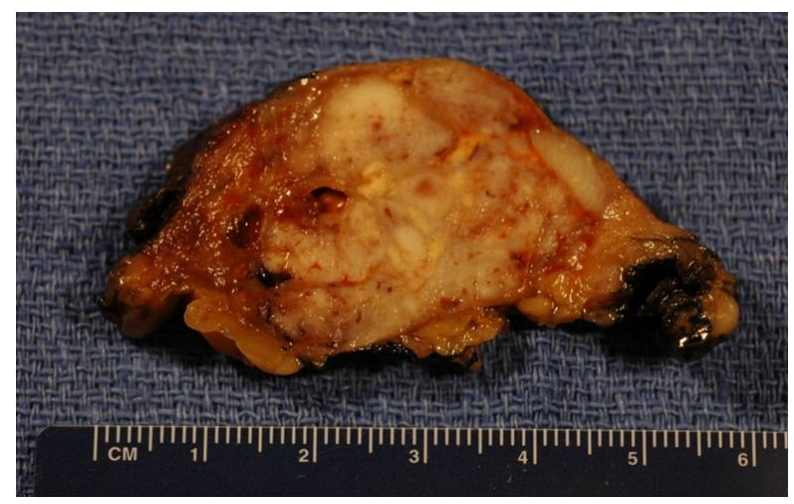

(a)

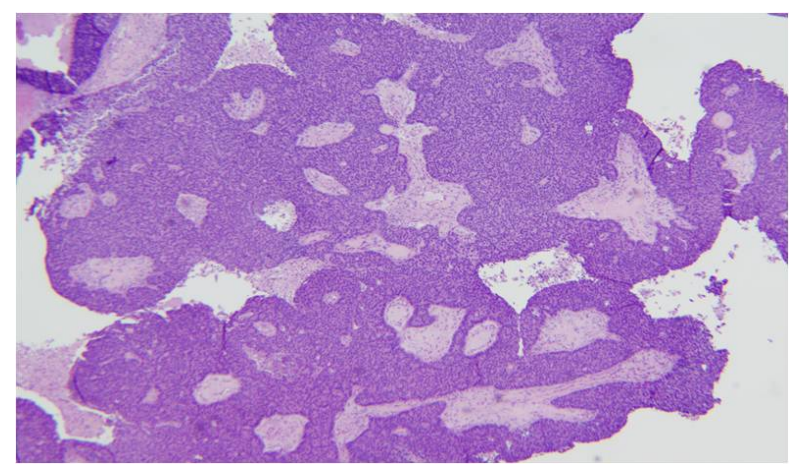

(b)

Figgure 2. (a) Cut gross section of completely resected thymic carcinoma; (b) Histopathologic examination $(\times 10)$ of the basaloid thymic carcinoma showing cells in a palisading arrangement.

uring $8.5 \mathrm{~cm} \times 6 \mathrm{~cm} \times 2 \mathrm{~cm}$ that was densely adherent to the pericardium and extending down over the right atrium (Figure 2(a)). The thymic fat and tissue were removed en bloc with a portion of pericardium to ensure complete resection margins. Histology demonstrated a basaloid thymic carcinoma, low grade, with no lymphovascular invasion (Figure 2(b)). All margins were negative, and lymph nodes included in the specimen was also negative for metastatic carcinoma. Postoperative course was uneventful. This tumor was classified as a World Health Organization (WHO) stage B3 or a Masoaka stage III. The patient received adjuvant radiation therapy totaling 60Gy. He remains tumor free after 36 months.

\section{Discussion}

Thymomas are the second most common tumors of the mediastinum (21\%) and the most common neoplasm of the anterosuperior mediastinum [1,2]. However, the incidence of thymic carcinomas is small and basaloid thymic carcinoma is one of the rarest variants. We found only 16 reported cases of basaloid thymic carcinomas in our search of PubMed database [5]. Thymic carcinomas are divided histologically into low-grade and high-grade tumors [2,3,6-8]. Basaloid carcinomas are low-grade tumors with a favorable prognosis compared to other forms of thymic carcinomas. Heterogeneity of thymomas has made classification of these tumors difficult. Several classification systems have been developed over the years; the most common classification systems used are the World Health Organization (WHO) system and the Masaoka system [2,3,8].

Patients usually have symptoms at presentation, and symptoms may be related to local mass effects causing chest pain, dyspnea, hemoptysis, cough, and the superior vena caval syndrome $[1,2,8]$. Most often the first diagnostic test after presentation is a chest X-ray, which is non-specific and will point to a mediastinal abnormality. Computed tomography is the modality of choice in diagnosis of thymomas. Magnetic resonance imaging is also frequently used, and has great value in surgical planning, especially if the tumor is invading adjacent structures based on initial imaging [2,8]. Positron emission tomography (PET) has emerged as a potential diagnostic tool to help distinguish a benign versus malignant neoplasm within the thymic gland; thus, potentially aiding in therapuetic management, such as dictating an intial operative approach versus biopsy [2,9]. Nonetheless, the utility of PET imaging for providing diagnostic acuracy for thymic carcinomas versus of malifnant neoplasms of the anterior mediatinum is still under evaluation $[2,9]$.

Fine needle aspiration is used by some to diagnose mediastinal tumors. However, many programs, including ours, do not use FNA routinely for concern of seeding of the tract or mediastinum with tumor cells [2,8]. A combination of blood tests for tumor markers, and testicular ultrasonography in men, can avoid uneccasary surgical intervention. In all other cases, diagnosis can be made at the time of surgical resection.

Surgery is the mainstay of treatment for thymomas with potential to cure. Our operative approach for anterior mediastinal masses is median sternotomy, as is favored by most for resecting thymic tumors, especially when thymic carcinoma is suspected $[2,8]$. The extent and completeness of the resection is an important factor in determining local recurrence and survival $[2,4,8]$.

Adjuvant radiation therapy remains controversial for completely resected Masaoka stage I and II tumors of the thymus. Adjuvant radiation has been shown to significantly increase 5-year survival rate (86\% VS. 48\%); especially in high risk Masaoka stage II tumors in the WHO categories of B2, B3, or C $[1,2,7,8]$. A number of studies have shown that changing radiation field or dose does not change survival [7]. Opinion in regards to adjuvant radiotherapy for Masaoka stage III thymomas is 
divided with some studies showing no significant survival difference between surgery alone and surgery with radiation $[4,8]$. However, other studies have demonstrated that adjuvant radiotherapy for advanced thymomas, particularly Masaoka stage III, has long-term benefits with complete or partial remission $[2,8]$.

Chemotherapy is not considered a treatment of choice when complete resection can be achieved [5]. However, cisplatin-based protocols have been used in patients with unresectable disease or gross residual disease [3]. Currently there are no standardized regimens.

In this case, we were able to achieve a complete resection of a basaloid thymic carcinoma, which as single therapy has a 5 year survival rate of $92.9 \%$ for a Masaoka stage III tumor [4]. Although the low-grade nature of basaloid thymic carcinoma, based on macroscopic invasion of the pericardium (Masaoka stage III) and histology features (WHO B3) in this case, adjuvant radiotherapy was added, and should enhance the overall favorable prognosis of this unusual thymic tumor.

\section{References}

[1] T. K. Varghese Jr. and C. L. Lau, “The Mediastinum,” In: C. M. Townsend Jr., R. D. Beauchamp, B. M. Evers, K. L. Mattox, Sabiston Text Book of Surgery, 18th Edition, Saunders, Philadelphia, 2008.

[2] V. Federico, A. Marco, D. Daniele, V. Domenico, A. R. Erino, G. de Tiziano, F. Federico and C. G. Furio, "Thymoma and Thymic Carcinoma," European Journal of Cardio-thoracic Surgery, Vol. 37, 2010, pp. 13-25.

[3] D. J. Kim, W. I. Yang, S. S. Choi, K. D. Kim and K. Y. Chung, "Prognostic and Clinical Relevance of the World
Health Organization Schema for the Classification of Thymic Epithelial Tumors: A Clinicopathologic Study of 108 Patients and Literature Review," Chest, Vol. 127, No. 3, 2005, pp. 755-761. doi:10.1378/chest.127.3.755

[4] K. Kondo and Y. Monden, “Therapy for Thymic Epithelial Tumors: A Clinical Study of 1320 Patients from Japan,” Annals of Thoracic Surgery, Vol. 76, No. 3, 2003, pp. 878-884. doi:10.1016/S0003-4975(03)00555-1

[5] P. J. Loehrer Sr., M. Jiroutek, S. Aisner, et al., “Combined Etoposide, Ifosfamide, and Cisplatin in the Treatment of Patients with Advanced Thymoma and Thymic Carcinoma: An Intergroup Trial,” Cancer, Vol. 91, No. 11, 2001, pp. 2010-2015. doi:10.1002/1097-0142(20010601)91:11<2010::AIDCNCR1226>3.3.CO;2-U

[6] Y. Morisaki, K. Takagi, S. Sano, et al., "Basaloid Carcinoma of the Thymus: Report of a Case,” Surgery Today, Vol. 36, No. 1, 2006, pp. 68-70. doi:10.1007/s00595-005-3072-x

[7] K. Ogawa, T. Uno, T. Toita, et al., "Postoperative Radiotherapy for Patients with Completely Resected Thymoma: A Multi-Institutional, Retrospective Review of $103 \mathrm{~Pa}-$ tients," Cancer, Vol. 94, No. 5, 2002, pp. 1405-1413. doi:10.1002/cncr.10373

[8] S. Tomaszek, D. A. Wigle, S. Keshavjee and S. Fischer, "Thymomas: Review of Current Clinical Practice," Annals of Thoracic Surgery, Vol. 87, No. 6, 2009, pp. 19731980. doi:10.1016/j.athoracsur.2008.12.095

[9] L. Luzzi, A. Campione, A. Gorla, G. Vassallo, A. Bianchi, A. Biggi and A. Terzi, "Role of Fluorine-Flurodeoxyglucose Positron Emission Tomography/Computed Tomography in Preoperative Assessment of Anterior Mediastinal Masses,” European Journal of Cardio-Thoracic Surgery, Vol. 36, No. 3, 2009, pp. 475-479. doi:10.1016/j.ejcts.2009.03.055 